\title{
Etiologic Spectrum and Follow-Up Results of Noninfectious Uveitis in Children: A Single Referral Center Experience
}

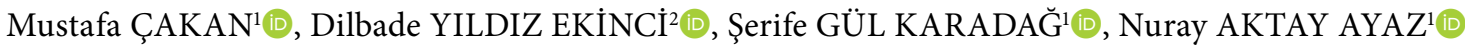 \\ ${ }^{1}$ Department of Pediatric Rheumatology, Kanuni Sultan Süleyman Training and Research Hospital, Istanbul, Turkey \\ ${ }^{2}$ Department of Ophthalmology, Kanuni Sultan Süleyman Training and Research Hospital, Istanbul, Turkey
}

\begin{abstract}
Objectives: This study aims to investigate the etiologic spectrum, demographic features, and long-term follow-up results of children with noninfectious uveitis (NIU).

Patients and methods: Files of patients with NIU were reviewed between May 2010 and September 2017. The cohort consisted of 54 juvenile uveitis patients (26 males, 28 females; mean age 7.7 years; interquartile range [IQR] 9.2 years) with 93 affected eyes. Location of uveitis, laterality, age at onset of uveitis, complications of uveitis, duration of follow-up, associated systemic diseases, pertinent laboratory tests, medications used, and status of uveitis at the time of enrollment were recorded from the files. All patients had final systemic and ocular examination at the last month of enrollment. Results: Twenty-seven patients (50.0\%) had juvenile idiopathic arthritis (JIA), 17 (31.4\%) had idiopathic uveitis, six (11.1\%) had Behçet disease (BD), and four (7.5\%) had tubulointerstitial nephritis and uveitis (TINU) syndrome. Median duration of follow-up for uveitis was 16 (IQR: 15) months. Anterior uveitis was seen in $81.4 \%$ of the patients $(65.9 \%$ had bilateral and $34.1 \%$ had unilateral anterior uveitis). Bilateral intermediate uveitis was observed in $11.2 \%$ and bilateral panuveitis in $7.4 \%$ of the patients. At the time of enrollment, 45 uveitis patients (83.3\%) were under remission. Complications of uveitis were observed in $18.5 \%$ of the patients.

Conclusion: Patients with JIA and BD should be regularly checked for uveitis. It is challenging to find an etiology in uveitis patients referred from ophthalmologists if initial questioning and examination do not reveal an overt rheumatologic disease. However, a simple urine test may help in establishing the diagnosis of TINU syndrome.

Keywords: Etiology; juvenile idiopathic arthritis; pediatrics; uveitis.
\end{abstract}

Uvea is the middle layer of the eye that is composed of the iris and ciliary body anteriorly and choroid posteriorly. Inflammation of the uvea is called uveitis which may be the component of a systemic disease or occur as an isolated process confined to eye. Uveitis in childhood is a rare entity and it is estimated that only $10-15 \%$ of all uveitis patients belong to the pediatric age group. ${ }^{1,2}$ Uveitis may be classified by anatomic location as anterior uveitis (iris and ciliary body), intermediate uveitis (vitreous and pars plana), posterior uveitis (choroid and retina), and panuveitis (all layers of uvea). ${ }^{3}$ The most common form of uveitis in pediatric age group is anterior uveitis. The symptoms of uveitis are pain, redness, excessive tearing, photophobia, floaters, and blurred vision. Unfortunately, most juvenile uveitis patients tend to be asymptomatic due to its silent and insidious course and diagnosed after structural complications develop. Also, children are less able to complain about subtle visual symptoms compared to adults. Furthermore, detailed ophthalmologic examination that may give important clues about the diagnosis and prognosis

Received: October 20, 2018 Accepted: November 20, 2018 Published online: January 28, 2019

Correspondence: Mustafa Çakan, MD. SBÜ Kanuni Sultan Süleyman Eğitim ve Araştırma Hastanesi Çocuk Romatolojisi Kliniği, 34303 Küçükçekmece, İstanbul, Turkey. Tel: +90 212 - 4041500 e-mail: mustafacakan@hotmail.com

\section{Citation:}

Çakan M, Yıldız Ekinci D, Gül Karadağ Ş, Aktay Ayaz N. Etiologic spectrum and follow-up results of noninfectious uveitis in children: a single referral center experience. Arch Rheumatol 2019;34(3):294-300. 
is extremely difficult in a non-cooperative young child that mostly depends on the patience and experience of the ophthalmologist in juvenile uveitis. ${ }^{1,2,4,5}$

The etiology of uveitis is broadly classified as infectious and noninfectious causes and masquerades of uveitis. Toxoplasmosis, toxocariasis, tuberculosis, and viral infections (e.g. herpes simplex virus or cytomegalovirus) are the most common etiologies in infectious uveitis. ${ }^{2,5}$ Masquerade syndromes such as leukemia, lymphoma, medulloepithelioma, and juvenile xanthogranuloma are rare disorders of childhood that may present with uveitis. ${ }^{2}$ The leading causes of juvenile noninfectious uveitis (NIU) are idiopathic uveitis, rheumatologic diseases (juvenile idiopathic arthritis [JIA], Behçet disease [BD], sarcoidosis including early-onset sarcoidosis and Blau syndrome, and vasculitides), tubulointerstitial nephritis and uveitis (TINU) syndrome, and VogtKoyanagi-Harada syndrome..$^{1,2,5}$

A stepladder approach is applied in the treatment of NIU. Local corticosteroids are the initial treatment in all patients. Short-term systemic corticosteroids and/or disease-modifying antirheumatic drugs (DMARDs) are indicated if local corticosteroids do not lead to remission. The introduction of biologics in rheumatology has improved the long-term results of children with rheumatic diseases and this is also applicable to NIU; it is recommended that biologics should not be delayed in children with active NIU and poor prognostic factors. ${ }^{4}$ In this study, we aimed to investigate the etiologic spectrum, demographic features, and long-term follow-up results of children with NIU.

\section{PATIENTS AND METHODS}

The files of patients with NIU were reviewed between May 2010 and September 2017 at Kanuni Sultan Süleyman Training and Research Hospital. The cohort consisted of 54 juvenile uveitis patients (26 males, 28 females; mean age 7.7 years; interquartile range [IQR] 9.2 years) with 93 affected eyes. Inclusion criteria were having a diagnosis of NIU, attending regularly to follow-up visits, being adherent to the medications, and completing the initial six months of follow-up. All JIA patients were diagnosed and classified according to the criteria of the International League of Associations for Rheumatology ${ }^{6}$ and uveitis was defined in accordance with the criteria of Standardization of Uveitis Nomenclature Working Group. ${ }^{3}$ All children were examined by the same ophthalmologist and thorough ocular examinations were performed including slit-lamp biomicroscopy, visual acuity check using Snellen charts or illiterate $\mathrm{E}$ charts, and intraocular pressure measurement by tonometer in each visit. Location of uveitis, laterality, age at onset of uveitis, complications of uveitis, duration of follow-up, associated systemic diseases, pertinent laboratory tests, medications used, and status of uveitis at the time of enrollment were recorded from the files. All patients had final systemic and ocular examinations at the last month of enrollment. The study protocol was approved by the Kanuni Sultan Süleyman Training and Research Hospital Ethics Committee. A written informed consent was obtained from the legal guardians of each patient. The study was conducted in accordance with the principles of the Declaration of Helsinki.

\section{Statistical analysis}

Shapiro-Wilk normality test was performed and quantitative data with non-normal distribution were presented as median and IQR. Categorical data were presented as counts and percentages. Statistical analyses were performed using the IBM SPSS version 22.0 software (IBM Corp., Armonk, NY, USA).

\section{RESULTS}

Median duration of follow-up for uveitis was 16 (IQR 15) months. Twenty-seven patients (50.0\%) had JIA, 17 (31.4\%) had idiopathic uveitis, six (11.1\%) had BD, and four (7.5\%) had TINU syndrome (Table 1).

Anterior uveitis was seen in $81.4 \%$ of the patients $(65.9 \%$ had bilateral and $34.1 \%$ had unilateral anterior uveitis). Bilateral intermediate uveitis was observed in $11.2 \%$ and bilateral panuveitis in $7.4 \%$ of the patients. Isolated posterior uveitis was not observed in any patient.

Juvenile idiopathic arthritis patients consisted of 21 (77.7\%) oligoarticular JIA (18 persistent and 3 extended oligoarticular JIA), two enthesitis-associated arthritis (ERA), 
Table 1. Demographic features of patients and most common anatomical locations of uveitis

\begin{tabular}{|c|c|c|c|c|c|c|c|c|}
\hline \multirow[t]{2}{*}{ Disease } & \multicolumn{2}{|c|}{ Number of patients } & \multicolumn{2}{|c|}{ Sex } & \multicolumn{2}{|c|}{$\begin{array}{l}\text { Age at diagnosis of uveitis } \\
\text { (year) }\end{array}$} & \multicolumn{2}{|c|}{$\begin{array}{c}\text { The most common anatomica } \\
\text { location of uveitis }\end{array}$} \\
\hline & $\mathrm{n}$ & $\%$ & Male & Female & Median & IQR & Anatomical location & $\%$ \\
\hline JIA & 27 & 50.0 & 12 & 15 & 4.8 & 2.5 & Bilateral anterior & 74.0 \\
\hline Idiopathic uveitis & 17 & 31.4 & 6 & 11 & 13.2 & 7.2 & Unilateral anterior & 35.2 \\
\hline Behçet disease & 6 & 11.1 & 5 & 1 & 14.3 & 4 & Bilateral panuveitis & 50.0 \\
\hline TINU & 4 & 7.5 & 3 & 1 & 13.4 & 8.4 & Bilateral anterior & 75.0 \\
\hline Total & 54 & & 26 & 28 & 7.7 & 9.2 & Anterior uveitis & 81.4 \\
\hline
\end{tabular}

two rheumatoid factor $(\mathrm{RF})$ negative polyarticular JIA, one psoriatic arthritis, and one systemic JIA. Five JIA patients had uveitis at the time of diagnosis of arthritis (4 persistent oligoarticular JIA and 1 RF negative polyarticular JIA). Only one patient had an initial diagnosis of uveitis and was later diagnosed as JIA (ERA). Median duration between diagnosis of JIA and uveitis was 23 (IQR 33) months.

Male and female ratio was close to each other in the whole cohort (26 males versus 28 females) and in JIA patients (12 males versus 15 females). While idiopathic uveitis patients $(6$ males versus 11 females) had female predominance, $\mathrm{BD}$ ( 5 males versus 1 female) and TINU syndrome ( 3 males versus 1 female) patients had male predominance. Median age at the time of diagnosis of uveitis was 7.7 (IQR 9.2) years in the whole cohort while this number was 4.8 (IQR 2.5) years in JIA patients, 13.2 (IQR 7.2) years in idiopathic uveitis patients, 13.4 (IQR 8.4) years in TINU syndrome patients, and 14.3 (IQR 4) years in BD patients.

Bilateral anterior uveitis was observed in $20(74.0 \%)$ JIA patients. Six JIA-associated uveitis patients had unilateral anterior uveitis and one patient had intermediate uveitis. Five patients had initially unilateral involvement but later had bilateral attacks. Anti-nuclear antibody (ANA) was positive in $90.4 \%$ of oligoarticular JIA-associated uveitis patients and $95.2 \%$ of oligoarticular JIA-associated uveitis patients were asymptomatic while uveitis was detected during routine screening.

Idiopathic uveitis patients had unilateral anterior uveitis $(n=6)$, bilateral anterior uveitis $(n=5)$, bilateral intermediate uveitis $(n=5)$, and bilateral panuveitis $(n=1)$. Five patients had granulomatous uveitis. None of the patients with idiopathic granulomatous uveitis had any sign or symptom of a systemic disease such as tuberculosis, sarcoidosis or multiple sclerosis that may present as granulomatous uveitis. All idiopathic uveitis patients were symptomatic, red eyes being the most common symptom. Three BD patients had bilateral panuveitis, two patients had unilateral, and one patient had bilateral anterior uveitis. Only one patient was asymptomatic. TINU syndrome patients had bilateral anterior uveitis $(n=3)$ and unilateral anterior uveitis $(n=1)$ and all of them had red eyes.

All patients with cells in the anterior chamber were treated with local corticosteroid drops (prednisolone acetate) and a short course of oral corticosteroids (prednisolone) was used in those with intermediate uveitis and panuveitis. Oral prednisolone was started at a dose of $2 \mathrm{mg} / \mathrm{kg} /$ day and used in full dose for two weeks and later tapered and discontinued in six-eight weeks depending on the severity of uveitis. All JIA-associated uveitis patients were treated with methotrexate and biologics were used in 14 patients of methotrexate-resistant JIA-associated uveitis (adalimumab in 11 and tocilizumab in 3 patients). In 12 JIA patients, biologics were used only for methotrexate-resistant uveitis. In three of 21 oligoJIA patients, uveitis developed while receiving etanercept that was being used for arthritis; etanercept was switched to adalimumab or tocilizumab. Methotrexate was used in nine patients (52.9\%) and adalimumab was used in one patient (5.8\%) of idiopathic uveitis. All six BD-associated uveitis patients were being treated with colchicine. Short-term systemic corticosteroids and azathioprine were used in four of them and cyclosporine in one. 
Half of $\mathrm{BD}$-associated uveitis patients were treated with biologics; two patients with interferon-alpha and one patient with adalimumab. Systemic treatment with methotrexate was needed in only one patient of TINU syndrome. Overall systemic corticosteroids were used in $40.7 \%$, DMARDs (methotrexate, azathioprine, and cyclosporine) in $68.5 \%$, and biologics in $33.3 \%$ of children with NIU. Biologics were started in patients who were unresponsive to local and/or systemic corticosteroids and DMARDs used for three months or earlier in some patients based on the decision of the ophthalmologist.

At the time of enrollment, 45 uveitis patients (83.3\%) were inactive while nine patients (5 idiopathic uveitis, 2 JIA, 1 BD, and 1 TINU syndrome) had active uveitis defined as having $\geq 0.5+$ cells in anterior chamber. All these patients had worsening activity of uveitis and methotrexate was initiated in six and biologics in three of them recently. Complications of uveitis were observed in 10 patients (18.5\%) such as temporary intraocular hypertension in five patients (3 JIA and 2 idiopathic uveitis), posterior synechia in two patients (1 JIA and 1 idiopathic uveitis), band keratopathy in two patients (1 JIA and 1 idiopathic uveitis), and decreased visual acuity in one patient (JIA).

\section{DISCUSSION}

Uveitis in childhood is a rare and challenging disorder. Early diagnosis and timely management result in prevention of complications. ${ }^{1,2,5}$ This study has shown that the most common anatomical location was the anterior region and oligoarticular JIA was the most commonly associated systemic disease in children with NIU.

Uveitis may be classified by anatomical location as anterior, intermediate, posterior, and panuveitis. Anterior uveitis is the most common type in pediatric age group in all ethnicities. The frequency of anterior uveitis has been reported to be $61.9 \%$ in the United States, ${ }^{7} 47.8 \%$ in Italy, ${ }^{8} 56.3 \%$ in Japan, ${ }^{9}$ and $40.7 \%$ in Iran. ${ }^{10}$ Our study also supported this finding with $81.4 \%$ of juvenile uveitis patients having anterior uveitis. The frequency of other uveitis types mostly depends on the geographical location. TugalTutkun ${ }^{5}$ compared the frequency of anatomical types from 18 studies conducted all over the world and concluded that the frequency of intermediate uveitis was $1.8-41.7 \%$, posterior uveitis was $5.5-26.6 \%$, and panuveitis was $1.8-34.4 \%$. The reason for the high ranges in different types of pediatric uveitis could be explained by whether the study was conducted by ophthalmologists or rheumatologists and whether the study included infectious causes of uveitis such as toxoplasmosis or toxocariasis that most commonly presents as posterior uveitis. The high number of anterior uveitis in our cohort could be explained by the high number of JIA patients that most commonly present as chronic bilateral anterior uveitis. The high frequency of acute anterior uveal involvement in TINU syndrome (100\%) and idiopathic uveitis (64.7\%) patients could also explain the high rate of anterior uveitis in this study. Exclusion of infectious causes may be the reason for the lack of any patient with isolated posterior uveal involvement.

Juvenile idiopathic uveitis is the most common systemic disease associated with uveitis in children. Risk factors for developing uveitis in JIA are female sex, having oligoarticular JIA, ANA positivity, and onset of arthritis before six years of age. Asymptomatic, chronic, and bilateral anterior uveitis is the most common presentation form of JIA-uveitis. ${ }^{4,5,7,8,11-13}$ This study also supported this notion that in all JIA patients, $77.7 \%$ had oligoarticular arthritis, $51.8 \%$ of them were females, $81.4 \%$ had positive ANA, and arthritis had begun before six years of age in $85.1 \%$. In $3-10 \%$ of JIA patients, uveitis may be diagnosed before arthritis., ${ }^{1,14}$ We had five patients of oligoarticular JIA-associated uveitis at the time of diagnosis of arthritis; in these five patients, uveitis had possibly started before the onset of arthritis but was missed due to the asymptomatic nature of the disease.

Idiopathic uveitis has been found to be the most common diagnosis in some pediatric uveitis series. Ferrara et al. ${ }^{7}$ reported that $51.4 \%$ of 286 children with uveitis had idiopathic uveitis while the study of Rahimi et al. ${ }^{10}$ showed that $63 \%$ of 54 juvenile uveitis patients had idiopathic uveitis. Pars planitis, which is defined as intermediate uveitis without an identifiable cause, is the leading diagnosis in idiopathic uveitis. Anterior uveitis is also a frequent presentation of idiopathic uveitis. ${ }^{1,2,5}$ Anterior uveitis was the most common 
form of idiopathic uveitis in this cohort (64.7\%) and $29.4 \%$ of idiopathic uveitis patients had pars planitis. Before identifying a patient as idiopathic uveitis, all possible etiologies must be discarded and the patient should be regularly checked for any evolving systemic disease. Detailed questioning and meticulous examination performed by ophthalmologists and pediatric rheumatologists are the key steps in revealing any possible clue for a systemic disease., ${ }^{1,5,11}$ To the best of our experience, ordering laboratory tests such as human leukocyte antigen-B51, serum angiotensin converting enzyme level or extractable nuclear antigen panel do not seem to contribute significantly in diagnosis since neither of these rheumatologic tests is specific nor pathognomonic for that disease if the patient does not have any clinical sign or symptom of that disease apart from uveitis. If a uveitis patient does not have any known systemic disease, the ruling out of infections should be directed by the ophthalmologist because most of the infectious causes have a suggestive ophthalmologic feature for that infectious agent. ${ }^{15}$ It has been recommended that tuberculosis should be ruled out in idiopathic uveitis patients before the initiation of systemic immunosuppressive treatment particularly in endemic countries. ${ }^{11,15}$ In our clinic; apart from complete blood count, urinalysis, blood chemistry and acute phase reactants, we order more specific tests depending on the physical examination and guidance of the ophthalmologist in patients with idiopathic uveitis.

Uveitis develops during the course of the disease in $70-80 \%$ of adult $\mathrm{BD}$ patients and the ratio of uveitis in pediatric BD is $27-80 \% .^{1,16}$ In $20 \%$ of the patients, uveitis may be the initial manifestation of the disease before the evolution of classical mucocutaneous lesions. ${ }^{1} \mathrm{BD}$ is highly endemic in Turkey and it was shown that it is the most common cause of systemic disease-associated uveitis in adults. ${ }^{17-19}$ Also, as demonstrated by the same studies, $\mathrm{BD}$-associated uveitis was the second most common cause of uveitis after JIA in Turkish children. ${ }^{17,18}$ The classical presentation of BD-associated uveitis is bilateral symptomatic panuveitis. The disease tends to follow a remitting and relapsing course. Males are more commonly and more severely affected than females in $\mathrm{BD}$-associated uveitis. Isolated anterior uveitis may be the only eye involvement in females. ${ }^{17,18}$
In this study, the one female patient with uveitis had unilateral anterior uveitis easily controlled with topical corticosteroids while the majority of the males had recurrent panuveitis attacks that needed biologic agent. Our small number of $\mathrm{BD}$-associated uveitis patients supports the literature indicating that boys with $\mathrm{BD}$ tend to have recurrent panuveitis attacks while girls with $\mathrm{BD}$ tend to have anterior uveitis. Due to the high prevalence of $\mathrm{BD}$ in our country, we follow-up each idiopathic uveitis patient for any evolving signs and symptoms of BD.

Tubulointerstitial nephritis and uveitis syndrome is an idiopathic disease characterized by manifestations of acute TINU. Renal disease is demonstrated as tubular dysfunction that is reflected as hematuria, proteinuria, and glycosuria in urine test. Uvea involvement is manifested mostly as acute symptomatic bilateral anterior uveitis. Uveitis may precede, but more commonly develops simultaneously at or after the onset nephritis. ${ }^{1,5,20,21}$ Kanno et al. ${ }^{22}$ reported five patients of TINU syndrome and uveitis and nephritis were diagnosed within one week from each other in all. We had four patients of TINU syndrome and all had renal manifestations as microscopic hematuria, glycosuria and mild proteinuria, and systemic manifestations. Two of the patients had simultaneous involvement of the eye and kidney, while proteinuria was detected one month before the onset of uveitis in one, and nephritis developed three weeks after the onset of uveitis in the other one. All of the patients had symptomatic anterior uveitis and uveitis was controlled by local corticosteroids except one who needed systemic corticosteroids and methotrexate. Jahnukainen et al. ${ }^{23}$ reported 12 patients of TINU syndrome, four of whom developed chronic anterior uveitis in the follow-up. They also stated that uveitis may develop later in the course of tubulointerstitial nephritis and recommended follow-up by an ophthalmologist in patients with acute tubulointerstitial nephritis for at least 12 months from diagnosis. The four TINU syndrome patients in our cohort were referred to pediatric rheumatology from ophthalmologists with the provisional diagnosis of idiopathic anterior uveitis for the search of systemic disease. Simple urine test and combination of abnormal urine manifestations with the uveitis enabled us to establish the diagnosis of TINU syndrome. 
We strongly recommend urine test to be the first laboratory test to be ordered in patients with idiopathic uveitis. Also, serial urine tests should be ordered in the next few months to check for any sign of renal involvement in patients with idiopathic anterior uveitis.

The aim of treatment in uveitis is suppression of ongoing inflammation and prevention of recurrences and complications. Topical or systemic corticosteroids are the first-line treatment options in children with NIU. DMARDs, methotrexate being the most commonly employed, should be initiated in patients unresponsive to corticosteroids and it is recommended that early initiation of immunosuppressive treatment should be considered in children with active uveitis with already established complications at the time of diagnosis., ${ }^{44-26}$ Complications of pediatric uveitis are band keratopathy, cataracts, synechia, glaucoma, cystoid macular edema, retinal detachment, neovascularization of retina, optic disc and iris, decreased visual acuity, and loss of vision. These complications have been reported to be seen in more than half of the patients. ${ }^{2,7,10}$ The study of Rosenberg et al. ${ }^{27}$ reported that $34 \%$ of pediatric uveitis patients had complications at the time of diagnosis and this number increased to $86.3 \%$ by three years after diagnosis. Delayed diagnosis, long duration of chronic inflammation due to late initiation of systemic immunosuppressive treatments, and already established complications at the time of diagnosis are considered as major risk factors for poor visual outcome..$^{1,2}$ We have observed either one of these complications in $18.5 \%$ of the patients. The low rate of uveitis complications in this study may be explained by the early detection of uveitis by strict screening program of patients with definite or suspected rheumatic diseases (JIA, BD, etc.), ease and comfort of working in the same institution with a dedicated ophthalmologist in pediatric uveitis, face-to-face discussions between the pediatric rheumatologists and ophthalmologist about treatment plans, and early initiation of immunosuppressives in children with difficult-to-treat uveitis.

Retrospective design is the major limitation of this study and also the data of a single center may not be enough to represent the true spectrum of noninfectious uveitis in the community.
In conclusion, children with any definite or suspected rheumatologic disease that may involve the eye should be regularly checked for uveitis. The results of this study have shown that JIA is the most commonly associated systemic disease with juvenile uveitis. We have seen that it is challenging to find an etiology in uveitis patients referred from ophthalmologists while a simple urine test may help in establishing a diagnosis of TINU syndrome and preventing unnecessary further workup. Effective communication between the rheumatologist and ophthalmologist is a sine qua non in the management of children with uveitis.

\section{Declaration of conflicting interests}

The authors declared no conflicts of interest with respect to the authorship and/or publication of this article.

\section{Funding}

The authors received no financial support for the research and/or authorship of this article.

\section{REFERENCES}

1. Reiff A, Kadayifcilar S, Özen S. Rheumatic inflammatory eye diseases of childhood. Rheum Dis Clin North Am 2013;39:801-32.

2. Chan NS, Choi J, Cheung CMG. Pediatric Uveitis. Asia Pac J Ophthalmol (Phila) 2018;7:192-9.

3. Jabs DA, Nussenblatt RB, Rosenbaum JT. Standardization of uveitis nomenclature for reporting clinical data. Results of the First International Workshop. Am $\mathrm{J}$ Ophthalmol 2005;140:509-16.

4. Constantin T, Foeldvari I, Anton J, de Boer J, Czitrom-Guillaume S, Edelsten C, et al. Consensusbased recommendations for the management of uveitis associated with juvenile idiopathic arthritis: the SHARE initiative. Ann Rheum Dis 2018;77:1107-17.

5. Tugal-Tutkun I. Pediatric uveitis. J Ophthalmic Vis Res 2011;6:259-69.

6. Petty RE, Southwood TR, Manners P, Baum J, Glass $\mathrm{DN}$, Goldenberg $\mathrm{J}$, et al. International League of Associations for Rheumatology classification of juvenile idiopathic arthritis: second revision, Edmonton, 2001. J Rheumatol 2004;31:390-2.

7. Ferrara M, Eggenschwiler L, Stephenson A, Montieth A, Nakhoul N, Araùjo-Miranda R, et al. The Challenge of Pediatric Uveitis: Tertiary Referral Center Experience in the United States. Ocul Immunol Inflamm 2018:1-8. 
8. Paroli MP, Spinucci G, Liverani M, Monte R, Pezzi PP. Uveitis in childhood: an Italian clinical and epidemiological study. Ocul Immunol Inflamm 2009; 17:238-42.

9. Keino H, Watanabe T, Taki W, Nakayama M, Nakamura T, Yan K, et al. Clinical features of uveitis in children and adolescents at a tertiary referral centre in Tokyo. Br J Ophthalmol 2017;101:406-10.

10. Rahimi M, Oustad M, Ashrafi A. Demographic and Clinical Features of Pediatric Uveitis at a Tertiary Referral Center in Iran. Middle East Afr J Ophthalmol 2016;23:237-40.

11. Majumder PD, Biswas J. Pediatric uveitis: An update. Oman J Ophthalmol 2013;6:140-50.

12. BenEzra D, Cohen E, Maftzir G. Uveitis in children and adolescents. Br J Ophthalmol 2005;89:444-8.

13. Tugal-Tutkun I, Quartier P, Bodaghi B. Disease of the year: juvenile idiopathic arthritis-associated uveitis-classification and diagnostic approach. Ocul Immunol Inflamm 2014;22:56-63.

14. Sen ES, Ramanan AV. Juvenile idiopathic arthritisassociated uveitis. Best Pract Res Clin Rheumatol 2017;31:517-34.

15. Rosenbaum JT, Dick AD. The Eyes Have it: A Rheumatologist's View of Uveitis. Arthritis Rheumatol 2018;70:1533-43.

16. Kramer M, Amer R, Mukamel M, Snir M, Jaouni T, Friling R. Uveitis in juvenile Behçet's disease: clinical course and visual outcome compared with adult patients. Eye (Lond) 2009;23:2034-41.

17. Kazokoglu H, Onal S, Tugal-Tutkun I, Mirza E, Akova Y, Ozyazgan Y, et al. Demographic and clinical features of uveitis in tertiary centers in Turkey. Ophthalmic Epidemiol 2008;15:285-93.

18. Yalçındağ FN, Özdal PC, Özyazgan Y, Batıoğlu F, TugalTutkun I. Demographic and Clinical Characteristics of Uveitis in Turkey: The First National Registry Report. Ocul Immunol Inflamm 2018;26:17-26.
19. Cakar Özdal MP, Yazici A, Tüfek M, Öztürk F. Epidemiology of uveitis in a referral hospital in Turkey. Turk J Med Sci 2014;44:337-42.

20. Alaygut D, Torun Bayram M, Ünlü M, Soylu A, Türkmen M, Kavukçu S. Acute tubulointerstitial nephritis-uveitis (TINU) syndrome developed secondary to paracetamol and codeine phosphate use: two case reports. Turk J Pediatr 2014;56:92-6.

21. Paladini A, Venturoli V, Mosconi G, Zambianchi L, Serra L, Valletta E. Tubulointerstitial nephritis and uveitis syndrome in a twelve-year-old girl. Case Rep Pediatr 2013;2013:652043.

22. Kanno H, Ishida K, Yamada W, Shiraki I, Murase $\mathrm{H}$, Yamagishi $\mathrm{Y}$, et al. Clinical and Genetic Features of Tubulointerstitial Nephritis and Uveitis Syndrome with Long-Term Follow-Up. J Ophthalmol 2018;2018:4586532.

23. Jahnukainen T, Ala-Houhala M, Karikoski R, Kataja J, Saarela V, Nuutinen M. Clinical outcome and occurrence of uveitis in children with idiopathic tubulointerstitial nephritis. Pediatr Nephrol 2011;26:291-9.

24. Sood AB, Angeles-Han ST. An Update on Treatment of Pediatric Chronic Non-Infectious Uveitis. Curr Treatm Opt Rheumatol 2017;3:1-16.

25. Henderson LA, Zurakowski D, Angeles-Han ST, Lasky A, Rabinovich CE, Lo MS. Medication use in juvenile uveitis patients enrolled in the Childhood Arthritis and Rheumatology Research Alliance Registry. Pediatr Rheumatol Online J 2016;14:9.

26. Cecchin V, Zannin ME, Ferrari D, Pontikaki I, Miserocchi E, Paroli MP, et al. Longterm Safety and Efficacy of Adalimumab and Infliximab for Uveitis Associated with Juvenile Idiopathic Arthritis. J Rheumatol 2018;45:1167-72.

27. Rosenberg KD, Feuer WJ, Davis JL. Ocular complications of pediatric uveitis. Ophthalmology 2004;111:2299-306. 